\title{
BMJ Open Prevalence and incidence of Parkinson's disease and other forms of parkinsonism in a cohort of elderly individuals in Southern Brazil: protocol for a population-based study
}

\author{
Gabriela Magalhães Pereira (D) ,, ${ }^{1,2}$ Nayron Medeiros Soares, ${ }^{1,2}$ \\ Neide Maria Bruscato, ${ }^{3}$ Emilio Hideyuki Moriguchi, ${ }^{4}$ João Senger, ${ }^{5}$ \\ Berenice Maria Werle, ${ }^{5}$ Rosa Maria Martins de Almeida, ${ }^{6}$ \\ Artur Francisco Schumacher-Schuh, ${ }^{2,7}$ Carlos Roberto de Mello Rieder ${ }^{8}$
}

To cite: Pereira GM, Soares NM, Bruscato NM, et al. Prevalence and incidence of Parkinson's disease and other forms of parkinsonism in a cohort of elderly individuals in Southern Brazil: protocol for a populationbased study. BMJ Open 2021;11:e054423. doi:10.1136/ bmjopen-2021-054423

- Prepublication history for this paper is available online. To view these files, please visit the journal online (http://dx.doi. org/10.1136/bmjopen-2021 054423).

Received 14 June 2021 Accepted 15 November 2021

D) Check for updates

(c) Author(s) (or their employer(s)) 2021. Re-use permitted under CC BY-NC. No commercial re-use. See rights and permissions. Published by BMJ.

For numbered affiliations see end of article.

\section{Correspondence to} Dr Gabriela Magalhães Pereira; gabrielamagalhaes.p@gmail. com

\section{ABSTRACT}

Introduction Parkinsonism is one of the most common neurological disorders affecting the elderly. Several population-based studies have determined the epidemiology of parkinsonism, mainly Parkinson's disease (PD), but there is still little evidence in the Brazilian population. This protocol study aims to assess the prevalence and incidence of cases of PD and other parkinsonian syndromes in a 5-year cohort in a population-based study in the southern region of Brazil. Methods and analysis A prospective populationbased longitudinal study, with a cohort of development of cases of parkinsonism, divided into two phases: in phase I, two questionnaires to screen for parkinsonism (Tanner's questionnaire), Rapid Eyes Movement (REM) sleep behaviour disorder (REM Sleep Behavior Disorder Single-Question Screen) and a short interview will be conducted with all elderly residents of Veranópolis (the first longevity Brazilian county located in the Rio Grande do Sul, Brazil) aged 60 or over. The positive screened cases will be examined independently by at least two movement disorder-trained physicians and prevalence will be determined. A comprehensive evaluation of prodromic symptoms, risk factors and clinical characteristics will be carried out. Subjects with subtle parkinsonism and a sample of healthy subjects will be followed for 5 years in a developmental cohort of parkinsonism cases. For crude incidence, all individuals admitted at the beginning of the study will be re-evaluated.

Ethics and dissemination The study was approved by the research ethics committee of the Hospital de Clínicas de Porto Alegre (protocol $n^{\circ} 4.095 .609$ ). All participants provide their informed consent before evaluations. Findings from this survey will be disseminated through peer-reviewed publications and will be presented at conferences.

\section{INTRODUCTION}

Parkinsonism is one of the most common neurological disorders that affects older
Strengths and limitations of this study

A strength of the prevalence study is the use of validated instruments for screening for parkinsonism and Rapid Eyes Movement Sleep Behavior Disorder.

- A key strength is the annually repeated measure design, which will facilitate the tracking of changes and appearance of symptoms over time.

- The follow-up of subtle cases in this study is interesting for investigating their natural history.

- The current study will be conducted in a city known for its longevity, which will allow for a wide range of age groups to be screened.

- The focus of the study on elderly people from a city in the southern region of Brazil is a limitation that can make it difficult to generalise to other Brazilian regions.

adults and is associated with the increased risk of adverse health outcomes and age-related brain pathologies. ${ }^{1}$ There are different types of parkinsonism, which can be primary such as Parkinson's disease (PD), or secondary to medication or other neurological conditions such as vascular and other neurodegenerative diseases. ${ }^{2}$

$\mathrm{PD}$ is a cause frequently identified as parkinsonism and has an estimated prevalence of $0.3 \%$ in industrialised countries for the entire population and may vary according to the age group, where, in people under the age of 60 , it ranges from $0.13 \%$ to $1.6 \%$ and reaches the maximum of $9 \%$ for individuals over 80 years old. ${ }^{3}$ Also, there is a slight predominance in men. ${ }^{4}$ The incidence is thought to be $15-17$ cases per 100000 person-years. ${ }^{56}$

The prevalence and incidence of PD and parkinsonism vary considerably in research 
reports, probably because of methodological differences, differences in case ascertainment, use of diagnostic criteria, geographical location, results of genetic and environmental factors and ethnic differences. ${ }^{67}$ However, based population studies have been estimating rates more concisely and with less variation due to the local geographic control and similar methodologies. ${ }^{8-10}$

To our knowledge, in Brazil, only two studies using this approach have determined the prevalence of parkinsonism. In the population-based study in Bambuí city (state of Minas Gerais), the prevalence was 3.3\% for PD among those over 64 years of age. ${ }^{7}$ For other parkinsonian syndromes, only one study conducted in the southwest of the country provides data on individuals over 75 years old and found a crude prevalence of $10.7 \%$ of parkinsonian syndromes in this population. ${ }^{11}$ Thereby, other Brazilian regions with different environmental and genetic characteristics lack studies on the epidemiology of parkinsonism. Veranópolis, a municipal district in the northeast of Rio Grande do Sul, is considered one of the cities of Brazil with high life expectancy. In the 90s, Veranópolis was recognised as the first longevity Brazilian county. Also, it was the first Brazilian city to receive the certificate of 'Friendly City for the Elderly' by the WHO. ${ }^{12}$ Life expectancy is 77.9 years for women and 73.2 years for men. ${ }^{13}$ This fact has been stimulated researchers to investigate determinants of longevity and several features' diseases related to ageing.

The ageing process is the most important risk factor to $\mathrm{PD},{ }^{6}$ and the differential diagnosis among parkinsonian syndromes is often difficult, based on the characterisation and evolution of clinical signs. Also, considering the prevalence and incidence of parkinsonism is little known in Brazil, and there is high longevity in Veranópolis, we propose this protocol study to assess the prevalence of cases of PD and other parkinsonian syndromes and to verify the conversion of prodromal symptoms to parkinsonian syndromes in a 5-year cohort in a population based of elderly in Veranópolis, Brazil.

\section{METHODS}

\section{Study design}

This protocol will be carried out in two distinct phases. Phase I (baseline survey) will be a cross-sectional, observational, population-based study, with the assessment of the prevalence of cases of PD and other parkinsonism. Phase II (incidence of parkinsonism) will be a prospective population-based longitudinal study, with the development cohort of cases of parkinsonism. In this phase, the participants who reach the end of phase I will be followed up annually for 5 years. Also, all participants contacted in phase I will be reassessed to determine the incidence of parkinsonism.

\section{Eligibility}

Eligible individuals are those $\geq$ aged 60 years, living in Veranópolis, Rio Grande do Sul, Brazil.

\section{Population and recruitment}

This study will be carried out with the participation of older people aged 60 or over, living in the county of Veranópolis, Rio Grande do Sul, Brazil and will be developed as an arm of the Veranópolis project: Studies on Aging, Longevity and Quality of Life, which addresses a large number of older people in the city.

According to the last census, the population of Veranópolis is estimated at 25936 inhabitants, where 3493 individuals are over 60 years old. ${ }^{14}$ Thus, our goal is to recruit all individuals over 60 years old of this city at the baseline survey to establish a census of the crude prevalence of PD and other parkinsonian syndromes. Initial recruitment started in August 2020 and the final recruitment is expected to occur in January 2023. Initially, participants have been invited to participate via advertisements in local radio programmes, where information about the study, the importance of this investigation and how they could be contacted have been provided. Two distinct methods are used to improve the recruitment of the baseline participants. First, we contact by phone call all individuals registered in the Veranópolis project. Second, a door-to-door approach will be conducted to assess the individuals registered in the health units or who cannot be reached by phone.

Those who screened positively showing clinical signs or symptoms in the second phase will be invited to participate in the 5-year cohort. A paired sample by age and sex that does not show any symptom will also be invited to participate in the cohort. They will be selected from negative screened in the first phase (figure 1). Matching will consider one control for two confirmed cases in the cohort study.

In addition, participants who obtain a clinical diagnosis of PD will be admitted to the cohort to monitor the progression of the disease.

\section{Patient and public involvement}

Patients and the public were not involved in the study's design, conduction, reporting or dissemination plans.

\section{Measures}

We chose our instruments for the cohort survey following the Movement Disorders Society (MDS) recommendation of the last update of criteria for Prodromal $\mathrm{PD}^{15}$ in order to evaluate potential predictive values of risk and prodromal markers in our individuals. Therefore, we also included additional domains relevant for the general clinical aspects and a screening questionnaire for phase I.

The instruments for data collection are divided into five classes: (1) clinical and sociodemographic screening; (2) parkinsonian syndrome screening; (3) prodromal screening, which includes the evaluation of non-motor symptoms such as (a) REM sleep behavioural disorder (RBD), (b) hyposmia, (c) constipation, (d) postural hypotension, (e) depressive symptoms, (f) excessive daytime somnolence, (g) global cognitive deficit, (h) aggressiveness and (i) loss of verbal fluency; (4) Quality of Life and 


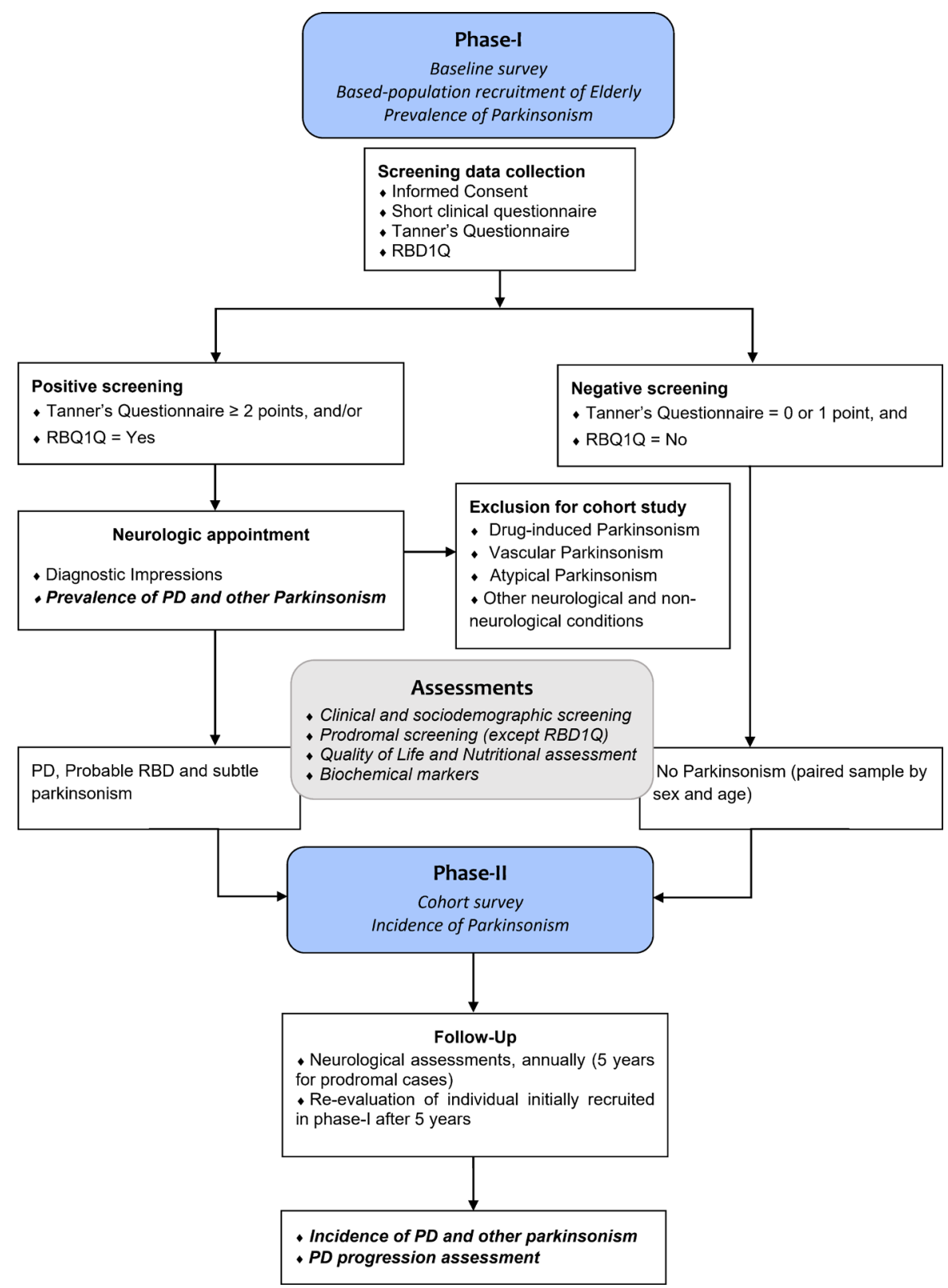

Figure 1 Flowchart of the study design. Prevalence of parkinsonism will be determinate in phase-I and, incidence of cases in phase-II. PD, Parkinson's disease; RBD1Q, REM Sleep Behavior Disorder Single-Question Screen.

Nutritional Assessment and (5) screening of biochemical markers. An overview of all the clinical aspects and instruments is presented in table 1 .

\section{Procedures}

We plan to execute a two-phase survey ascertainment approach to verify the prevalence (phase I, the baseline survey) and incidence of cases (phase II, the cohort survey). A flowchart of the procedures in each phase is presented in figure 1.

Baseline survey-investigating the prevalence of parkinsonian syndromes and non-motor symptoms associated with PD

In phase I, a cross-sectional study will be carried out in the first step to assess the prevalence of parkinsonian syndromes. Trained interviewers will contact by phone or in their residence in Veranópolis and apply
Tanner's questionnaire, RBDQ1 (items 2 and 3a), and questions that address the following aspects: age, sex, coffee consumption and smoking habits. Interviews are occurring daily by phone and presential door-todoor is expected to occur once a week. Those who present positive scores for motor or non-motor symptoms will be referred for neurological evaluation. These individuals will perform a neurological evaluation using MDS-Unified Parkinson's Disease Rating Scale (MDS-UPDRS). During the consultation, individuals will be evaluated by two movement disordertrained neurologists. A senior neurologist expert in movement disorders will evaluate all suspect cases to confirm the diagnosis and etiologic classification of parkinsonism. Presential consultations will occur monthly at Moriguchi Institute. In each case, we 
Table 1 Measures and instruments of the survey

\section{Construct}

Clinical and

sociodemographic

screening

Parkinsonian syndrome

screening

\section{Measure}

Age, sex, education, occupation, family history of parkinsonism, diabetes, physical activity, smoking, tea and coffee consumption, exposure to pesticides and heavy metals, comorbidities, medications in use, and history of falls.

The Tanner's questionnaire adapted by Barbosa et al, ${ }^{7}$ consists of nine objective and easily interpreted items about changes and difficulties in motor function. A cut-off score of $\geq 2$ will be used, whose sensitivity and specificity for the diagnosis of parkinsonism is $100 \%$ and $29 \%$, respectively.

\section{Prodromal screening}

REM Sleep Behavior

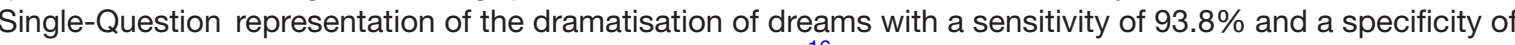

Screen-RBD1Q

REM Sleep Behavior

Disorder Screening

Questionnaire-Brazilian

version

Sniffin' Sticks odor

identification test (SST-

Burghart Medizintchnik,

Germany)

Constipation

Postural hypotension

A 13-item, self-administered, dichotomous instrument, which may be useful for RBD screening and diagnosis of Brazilian patients with Parkinson's disease (PD). ${ }^{17}$

It consists of 16 pens with the common odorants: orange, rose, banana, cinnamon, peppermint, licorice, garlic, coffee, leather, apple, cloves, pineapple, anise, fish, lemon and turpentine (strong pine aroma). During the test, the pen is placed $2 \mathrm{~cm}$ from the nostrils of the patient, who needs each pen is at least $30 \mathrm{s.}{ }^{18}$

\section{Screening about usual daily bowel movement frequency and the use of laxatives.}

Assessment of blood pressure and heart rate in the supine position (period in supine position to identify the odour and choose one of the four options listed for each pen. The interval between before the measurement is at least $5 \mathrm{~min}$ ) and for up to $3 \mathrm{~min}$ while upright. A positive test shows a decrease in systolic blood pressure (BP) $\geq 20 \mathrm{~mm} \mathrm{Hg}$ and/or in diastolic BP $\geq 10 \mathrm{~mm} \mathrm{Hg}$ changing from supine to upright position. ${ }^{19}$ If the test is negative, the passive head-up tilt testing is recommending. ${ }^{20}$

Beck Depression Inventory-II

It consists of 21 items, ranging in intensity from 0 to 3 , related to depressive symptoms. A good cut-off of $13 / 14$ has been suggested in PD. ${ }^{21}$

Epworth Sleepiness

Scale

It is a self-administered questionnaire that refers to the possibility of napping in eight everyday situations. To scale the probability of napping, a scale from 0 to 3 is used, where 0 corresponds to none and three represents the high probability of napping. Scores $>10$ points indicate a great possibility of Excessive Daytime Sleepiness and scores $>16$ are indicative of severe sleepiness. ${ }^{22}$

Montreal Cognitive Assessement

State-Trait Anger Expression Inventory-2 ${ }^{24}$

It is a brief screening for mild cognitive decline that evaluates executive functions, visuospatial skills, naming, memory recovery, digits, sentence, abstract reasoning and orientation. ${ }^{23}$

It is an instrument that provides concise measures of the experience and expression of anger.

It makes it possible to investigate not only the intensity of angry feelings but the frequency with which they are experienced. It contains 57 items that assess the state of anger, traits of anger and expression and anger control, with an average application time of 12-15 min.

Verbal fluency test (FAS) In semantic FAS, subjects are asked to speak the largest number of animals for 1 min. In phonemic FAS, the subjects will be asked to generate the largest number of words starting with / $F$ / - / A / - / / for 1 min, respectively. ${ }^{25} 26$

MDS-Unified

Assessment Scale for

Parkinson's Disease

(MDS-UPDRS)

The MDS-UPDRS is an instrument to assess the progression and effectiveness of treatments in PD. ${ }^{27}$ It consists of 42 items, classified into four parts: part I (non-motor aspects of daily life), part II (motor aspects of daily life), part III (motor evaluation) and part IV (motor complications). Prodromal criteria recommend a MDS-UPDRS score $>6$ for possible subthreshold parkinsonism, excluding postural and action tremor and possible confounders. ${ }^{1528}$

Quality of Life and Nutritional Assessment

WHO Quality of Life- It consists of 24 questions, which vary on a scale of 1 to 5 points. The questions are related to OLD sensory abilities; autonomy; past, present, and future activities; social participation; death and dying; and intimacy. Higher scores represent good quality of life and lower scores represent a low quality of life. ${ }^{29}$

Food record A food record will be applied for three alternating days of the week. The individual reports all the food and drinks consumed, including the food consumed outside the home. 
Table 1 Continued

\begin{tabular}{ll}
\hline Construct & Measure \\
\hline $\begin{array}{l}\text { Mini Nutritional } \\
\text { Assessment }\end{array}$ & $\begin{array}{l}\text { It is a nutritional screening and assessment tool that can identify geriatric patients who are } \\
\text { malnourished or at risk of malnutrition. Scores from } 12 \text { to } 14 \text { indicate normal nutritional status, } \\
\text { 8-11 at risk of malnutrition and 0-7 malnutrition. }\end{array}$ \\
$\begin{array}{l}\text { Alcohol Use Disorder } \\
\text { Identification Test }\end{array}$ & $\begin{array}{l}\text { The instrument consists of 10 items covering three domains: 1) Frequency of alcohol consumption, } \\
\text { 2) dependence on consumption and 3) negative consequences of alcohol consumption. A score } \\
\text { of } 0 \text { to } 7 \text { indicates a lower risk of consumption; } 8 \text { to } 15 \text { points indicates a risky use; } 16 \text { to 19 points } \\
\text { indicate a harmful use, and 20 or more points indicates a probable dependence. }\end{array}$ \\
$\begin{array}{l}\text { Screening of biochemical } \\
\text { markers }\end{array}$ & $\begin{array}{l}\text { Complete blood count, platelets, glucose, total cholesterol, high-density lipoprotein and low- } \\
\text { density lipoprotein cholesterol, creatinine, urea, total bilirubin and fractions, triglycerides, ultra- } \\
\text { sensitive C reactive protein, sodium, potassium, serum cholinesterase, vitamin D, vitamin B12, } \\
\text { transferrin, ferritin, iron and uric acid. }\end{array}$ \\
\hline
\end{tabular}

attempt to provide a cause of parkinsonism according to current diagnosis criteria (table 2).

If participants have not received a definitive diagnosis after neurological assessment, then they will receive one of the diagnostic impressions according to the following conditions:

1. Diagnosis of Clinically Established Probable PD.

2. Diagnosis of Clinically Established PD.

3. Atypical parkinsonism.

4. Secondary parkinsonism.

5. Motor symptoms secondary to another neurological condition (non-parkinsonian condition, eg, stroke, amyotrophic lateral sclerosis, etc).

6. Motor symptoms secondary to a condition other than central nervous system (CNS) disease (osteoarthritis, musculoskeletal injuries, etc).

7. Presence of RBD with no motor symptoms.

8. Presence of subtle parkinsonism without diagnostic criteria for the conditions above.

Once a neurological condition is identified, the individual will receive guidance from the neurologist and professional team on the available treatments.
After performing the diagnostic impression, individuals classified in groups A, B, G and $\mathrm{H}$ and a sample of asymptomatic individuals will respond to the instruments corresponding to the complete clinical and sociodemographic questionnaire, prodromal and risk markers, quality of life and nutritional assessment. During the appointment, a blood sample will be collected for the analysis of biomarkers. Sample processing will occur in two different forms. Samples for routine exams will be processed at the local laboratory unit that serves the city community, following the processing protocols of the biochemical unit. To measure the levels of transferrin, ferritin, iron and uric acid, a blood collection will be made with a total volume of $10 \mathrm{~mL}$ of venous blood from the patients. The samples will be placed in tubes without anticoagulants and centrifuged. The plasma serum will be frozen at a temperature of $-80^{\circ} \mathrm{C}$ until analysis. The serum levels of the evaluated markers will be measured using ELISA kits (ELISA method), using commercial reagents according to the manufacturer's instructions.

Table 2 Definition of parkinsonism causes according to current diagnosis criteria

\begin{tabular}{|c|c|}
\hline Parkinsonism & Diagnosis criteria \\
\hline Idiopathic PD & United Kingdom Brain Bank Criteria. ${ }^{33-35}$ Other causes should have been excluded. \\
\hline Multiple system atrophy & Consensus Statement. ${ }^{36}$ \\
\hline Dementia with Lewy bodies. & $\begin{array}{l}\text { Consensus guidelines for the clinical and pathological diagnosis of dementia with Lewy } \\
\text { bodies. }\end{array}$ \\
\hline Vascular parkinsonism & $\begin{array}{l}\text { Presence at least two of these clinical conditions: repeated strokes with abrupt onset } \\
\text { (with the progression of parkinsonism signs), pyramidal signs, broad-based rigid gait, and } \\
\text { hypertension. Also, imaging exams presenting lesions in white matter, in basal ganglia or } \\
\text { widespread in both hemispheres. }{ }^{41}\end{array}$ \\
\hline
\end{tabular}

NINDS-SPSP, National Institute of Neurological Disorders and Stroke and the Society for Progressive supranuclear palsy; PD, Parkinson's disease. 
Cohort survey-investigation of the incidence of parkinsonian syndromes and the risk of converting associated non-motor symptoms to PD

Participants who have reached the end of phase I will be invited to participate in the cohort. However, groups C-F will be excluded from this phase once they have a specific diagnosis according to the consensus criteria or have no suspicion of prodromal symptoms. Thus, participants with primary PD diagnosis, changes suggestive of RBD without motor changes, the presence of subtle parkinsonism without diagnostic criteria for the above conditions (groups A-B and $\mathrm{G}-\mathrm{H}$ ) and the control sample will be followed up annually up to complete 5 years.

Annually, neurological evaluations will be performed, including the assessments using the same instruments and blood collection samples as in phase I. We plan to perform five consecutive collections from the completion of phase I. The screening questionnaires will be applied in controls to verify the development of parkinsonism syndromes in this group. In the case of positive screening in this group, the participant will receive the same orientations and follow-up as the other positive parkinsonian participant. The expected follow-up period is 5 years.

In addition, to verify the new cases of parkinsonism in the total elderly population of Veranópolis, all participants screened at the beginning of the study will be contacted and evaluated after 5 years.

\section{Analytical approach}

Initially, survey data will be stored in the Excel programme from the Microsoft Office professional plus 2019. For each instrument, we will follow all recommended methods of summarisation and presentation of data. We will monitor missing data according to the number of participants admitted during the data collection and take proactive measures to minimise it in future collections. We plan to use for minor missing data a multiple imputation method, where the imputed items are used to obtain punctual estimates.

For quantitative analyses, we will export data to IBM SPSS Statistics for Windows V.26. Quantitative variables will be assessed to verify their distribution characteristics. In case of asymmetry, non-parametric tests, transformation methods of data or alternative methods (eg, qualitative analysis) will be used appropriately if necessary. Descriptive analysis will characterise participants' responses or scores using mean, the SD of mean, median, minimum and maximum or percentage, according to the variable type and considering the distribution analysis. For comparisons, we will use the $\chi^{2}$ test for categorical variables and $t$ test for continuous variables, such as sociodemographic status, clinical assessments and biomarkers. Non-parametric tests will be appropriately used when necessary.

The cross-sectional survey will obtain prevalence rates for all types of parkinsonism found and for specific aetiologies, according to age and sex, using the $\chi^{2}$ test. For the association between risk factors, multiple linear or logistic regression will be performed for continuous outcomes or Poisson regression with robust variances to analyse qualitative variables.

In the cohort survey, we will employ Cox proportional hazard models to determine the number of participants with the development of PD or another parkinsonism type. Also, a generalised linear mixed model or mixed logistic regression will be used for repeated measures. All associations will be first assessed using univariate models. After, we will include specific covariates in each model according to the clinical relevance associated with the outcome of interest in the multivariate analysis. Time variable will be an important continuous predictor in the models (being coded as 0 months $=0,12$ months $=1$, 2 years $=2$, 3 years $=3,4$ years $=4$ and 5 years $=5$ ). In all analyses, the significance level corresponding to $\mathrm{p}<0.05$ will be considered.

\section{RECRUITMENT PROGRESS}

We started the recruitment of phase I in August 2020. Until now, 766 older people were partially screened and presented a mean age of $73.00 \pm 8.33$ years, schooling of $8.64 \pm 9.66$ years of study and $59 \%$ (452) were women. Regarding smoking habits, $73.5 \%$ never smoked, $23.8 \%$ reported previous smoking habits and only $2.7 \%$ currently smoke. The initial screening for parkinsonism, according to Tanner's Questionnaire, 20.7\% had a positive outcome. RBD1Q showed $10.6 \%$ of individuals had a positive screening for REM sleep disorders. Thus, of the sample collected, $25.4 \%$ were considered able to perform the neurological appointment. Phase I is in progress only by phone call due to the COVID-19 pandemic. We estimate to start the neurological assessment in July 2021, following all preventive and health measures provided by local health authorities and WHO.

\section{ETHICS AND DISSEMINATION}

The research ethics committee of the Hospital de Clínicas de Porto Alegre (HCPA) Brazil (protocol $\mathrm{n}^{\circ}$ 4.095.609) approved the study. All participants provide their informed consent before evaluations. The findings of this survey will be disseminated through peer-reviewed publications and will be presented at conferences.

\section{Author affiliations}

${ }^{1}$ Programa de Pós-Graduação em Ciências Médicas, Faculdade de Medicina, Universidade Federal do Rio Grande do Sul, Porto Alegre, Rio Grande do Sul, Brazil

${ }^{2}$ Serviço de Neurologia, Hospital de Clínicas de Porto Alegre, Porto Alegre, Rio Grande do Sul, Brazil

${ }^{3}$ Coordenação de Pesquisa, Associação Veranense de Assistência em Saúde/AVAES, Veranópolis, Brazil

${ }^{4}$ Departmento de Medicina Interna, Universidade Federal do Rio Grande do Sul, Porto Alegre, Rio Grande do Sul, Brazil

${ }^{5}$ Centro de Estudos do Envelhecimento, Instituto Moriguchi, Veranópolis, Brazil ${ }^{6}$ Instituto de Psicologia, Universidade Federal do Rio Grande do Sul, Porto Alegre, Rio Grande do Sul, Brazil

${ }^{7}$ Departamento de Farmacologia, Universidade Federal do Rio Grande do Sul, Porto Alegre, Rio Grande do Sul, Brazil 
${ }^{8}$ Departamento de Clínica Médica, Universidade Federal de Ciencias da Saude de Porto Alegre, Porto Alegre, Rio Grande do Sul, Brazil

Acknowledgements The authors acknowledge the city hall of Veranópolis and Moriguchi Institute and the team for their support in the set-up of this study.

Contributors GMP and CRdMR conceived the original study, design of the work and drafting the work. GMP, NMS, AFS-S, RMMdA and CRdMR revising it critically for important intellectual content and are accountable for all aspects of the work. GMP, CRdMR, NMS, AFS-S, RMMdA, NMB, JS, EHM and BMW provided the final approval of the version to be published.

Funding This work was supported in part by the Coordination for the Improvement of Higher Education Personnel (CAPES)—Finance Code 001. Award/grant number: N/A.

Competing interests None declared.

Patient and public involvement Patients and/or the public were not involved in the design, or conduct, or reporting, or dissemination plans of this research.

Patient consent for publication Not applicable.

Provenance and peer review Not commissioned; externally peer reviewed.

Open access This is an open access article distributed in accordance with the Creative Commons Attribution Non Commercial (CC BY-NC 4.0) license, which permits others to distribute, remix, adapt, build upon this work non-commercially, and license their derivative works on different terms, provided the original work is properly cited, appropriate credit is given, any changes made indicated, and the use is non-commercial. See: http://creativecommons.org/licenses/by-nc/4.0/.

\section{ORCID iD}

Gabriela Magalhães Pereira http://orcid.org/0000-0003-0284-6678

\section{REFERENCES}

1 Buchman AS, Wilson RS, Shulman JM, et al. Parkinsonism in older adults and its association with adverse health outcomes and neuropathology. J Gerontol A Biol Sci Med Sci 2016;71:549-56.

2 McFarland NR. Diagnostic approach to atypical parkinsonian syndromes. Continuum 2016;22:1117-42.

3 Nerius M, Fink A, Doblhammer G. Parkinson's disease in Germany: prevalence and incidence based on health claims data. Acta Neurol Scand 2017;136:386-92.

4 Capriotti T, Terzakis K. Parkinson disease. Home Healthc Now 2016;34:300-7

5 Twelves D, Perkins KSM, Counsell C. Systematic review of incidence studies of Parkinson's disease. Mov Disord 2003;18:19-31.

6 Tysnes O-B, Storstein A. Epidemiology of Parkinson's disease. $J$ Neural Transm 2017;124:901-5.

7 Barbosa MT, Caramelli P, Maia DP, et al. Parkinsonism and Parkinson's disease in the elderly: A community-based survey in Brazil (the Bambuí study). Movement Disorders 2006;21:800-8.

8 de Rijk MC, Tzourio C, Breteler MMB. Prevalence of parkinsonism and Parkinson's disease in Europe: The EUROPARKINSON collaborative study. J Neurol Neurosurg Psychiatry 1997;62.

9 Chan DK, Dunne M, Wong A, et al. Pilot study of prevalence of Parkinson's disease in Australia. Neuroepidemiology 2001;20:112-7.

10 Zhang Z-X, Anderson DW, Huang J-B, bin HJ, et al. Prevalence of Parkinson's disease and related disorders in the elderly population of greater Beijing, China. Movement Disorders 2003;18:764-72.

11 Vale TC, Barbosa MT, Resende EdePF, et al. Parkinsonism in a population-based study of individuals aged 75+ years: the Pietà study. Parkinsonism Relat Disord 2018;56:76-81.

12 World Health Organization. Veranópolis - Age-Friendly World, 2016. Available: https://extranet.who.int/agefriendlyworld/network/ veranopolis/

13 Senger J, Senger JE, Bruscato NM. Characterisation of vitamin B12 level and its relationship with cognitive performance in the community-dwelling elderly. Nutr Food Sci 2020;3:132-9.

14 Censo. IBGE Censo 2010. Instituto Brasileiro de Geografia E Estatística, 2010. Available: http://censo2010.ibge.gov.br/

15 Heinzel S, Berg D, Gasser T, et al. Update of the MDS research criteria for prodromal Parkinson's disease. Movement Disorders 2019;34:1464-70.
16 Postuma RB, Arnulf I, Hogl B, et al. A single-question screen for rapid eye movement sleep behavior disorder: a multicenter validation study. Mov Disord 2012;27:913-6.

17 Pena-Pereira MA, Sobreira-Neto MA, Sobreira E, et al. Validation of the Brazilian Portuguese version of the rapid eye movement sleep behavior disorder screening questionnaire (RBDSQ-BR). Arq Neuropsiquiatr 2020;78:629-37.

18 Santin R, Fonseca VF, Bleil CB, et al. Olfactory function and Parkinson's disease in southern Brazil. Arq Neuropsiquiatr 2010;68:252-7.

19 Schatz IJ, Bannister R, Freeman RL. Consensus statement on the definition of orthostatic hypotension, pure autonomic failure, and multiple system atrophy. Neurology 1996;46.

20 Lahrmann $\mathrm{H}$, Cortelli P, Hilz M, et al. EFNS guidelines on the diagnosis and management of orthostatic hypotension. Eur $J$ Neurol 2006;13:930-6.

21 Visser M, Leentjens AFG, Marinus J, et al. Reliability and validity of the Beck depression inventory in patients with Parkinson's disease. Mov Disord 2006;21:668-72.

22 Johns MW. A new method for measuring daytime sleepiness: the Epworth Sleepiness scale. Sleep 1991;14:540-5.

23 Nasreddine ZS, Phillips NA, Bédirian V, et al. The Montreal cognitive assessment, MoCA: a brief screening tool for mild cognitive impairment. J Am Geriatr Soc 2005;53:695-9.

24 Spielberger CD. Staxi-2: state-trait anger expression inventory-2. In: Professional manual. FL: Psychological Assessment Resources, 1999.

25 Machado TH, Fichman HC, Santos EL, et al. Normative data for healthy elderly on the phonemic verbal fluency task - FAS. Dement Neuropsychol 2009;3:55-60.

26 Opasso PR, Barreto SDS, Ortiz KZ. Phonemic verbal fluency task in adults with high-level literacy. Einstein 2016;14:398-402.

27 Goetz CG, Tilley BC, Shaftman SR, et al. Movement Disorder Society-sponsored revision of the Unified Parkinson's Disease Rating Scale (MDS-UPDRS): Scale presentation and clinimetric testing results. Movement Disorders 2008;23:2129-70.

28 Berg D, Postuma RB, Adler $\mathrm{CH}$, et al. MDS research criteria for prodromal Parkinson's disease. Mov Disord. 2015;30:1600-11.

29 WHOQOL-OLD Group. World Health Organization quality of Life-old (WHOQOL-OLD). In: The WHOQOL-OLD manual, 2006.

30 Guigoz Y. The Mini Nutritional Assessment (MNA) review of the literature--What does it tell us? J Nutr Health Aging 2006;10:466-87.

31 Lima CT, Freire ACC, Silva APB, et al. Concurrent and construct validity of the audit in an urban Brazilian sample. Alcohol Alcohol 2005;40:584-9.

32 dos Santos WS, Gouveia VV, Fernandes DP. Alcohol use disorder identification test (audit): exploring its psychometric parameters. J Bras Psiquiatr 2012;61.

33 Hughes AJ, Ben-Shlomo Y, Daniel SE. What features improve the accuracy of clinical diagnosis in parkinson's disease: A clinicopathologic study. Neurology2001;57.

34 Hughes AJ, Daniel SE, Kilford L, et al. Accuracy of clinical diagnosis of idiopathic Parkinson's disease: a clinico-pathological study of 100 cases. J Neurol Neurosurg Psychiatry 1992;55:181-4.

35 Hughes AJ, Daniel SE, Lees AJ. Improved accuracy of clinica diagnosis of Lewy body Parkinson's disease. Neurology 2001;57:1497-9.

36 Gilman S, Low PA, Quinn N, et al. Consensus statement on the diagnosis of multiple system atrophy. J Neurol Sci 1999;163:94-8.

37 Litvan I, Agid Y, Goetz C, et al. Accuracy of the clinical diagnosis of corticobasal degeneration: a clinicopathologic study. Neurology 1997;48:119-25.

38 McKeith IG, Galasko D, Kosaka K, et al. Consensus guidelines for the clinical and pathologic diagnosis of dementia with Lewy bodies (DLB): report of the Consortium on DLB International workshop. Neurology 1996;47:1113-24.

39 Litvan I, Agid Y, Jankovic J, et al. Accuracy of clinical criteria for the diagnosis of progressive supranuclear palsy (Steele-RichardsonOlszewski syndrome). Neurology 1996;46:922-30.

40 Jiménez-Jiménez FJ, Ortí-Pareja M, Ayuso-Peralta L, et al. Druginduced parkinsonism in a movement disorders unit: a four-year survey. Parkinsonism Relat Disord 1996;2:145-9.

41 Foltynie T, Barker R, Brayne C. Vascular parkinsonism: a review of the precision and frequency of the diagnosis. Neuroepidemiology 2002;21:1:1-7 